\title{
Nach Schaumsklerosierung bei Varikosis Kompressionsstrümpfe mit höheren Drücken?
}

Cavezzi A et al. Compression with $23 \mathrm{mmHg}$ or $35 \mathrm{mmHg}$ stockings after saphenous catheter foam sclerotherapy and Phlebectomy of varicose veins: A

randomized controlled study. Phlebology 2019; 34: 98 - 106

Die postoperative Kompression nach Therapie der Varikosis wurde in zahlreichen Studien untersucht. Endgültige Schlussfolgerungen zur Effizienz, Sicherheit, Dauer und zum notwendigen Druck ergaben sich bisher nicht. Die Studie untersuchte die Ergebnisse der Anwendung von 2 verschiedenen Kompressionsstrümpfen in der unmittelbaren und frühen postoperativen Periode nach kathetergestützter Schaumsklerosierungstherapie.

In die italienische randomisierte kontrollierte Einfachblindstudie wurden $94 \mathrm{~Pa}$ tient/-innen (59 Frauen, 35 Männer) im mittleren Alter von 53,5 Jahren aufgenommen. An ihnen wurden 97 Beine mit kathetergestützter Schaumsklerosierungstherapie und Phlebektomie behandelt. Nach der Voruntersuchung vor Beginn der Therapie wurden die Patient/-innen randomisiert für die postoperative Therapie mit 2 verschiedenen Kompressionsstrümpfen. Gruppe $A$ ( $n=48$ Beine) wurde mit dem Struva $23{ }^{\circledR}$ (Medi GmbH, Bayreuth) behandelt, der einen Druck von 23 mmHg an Knöcheln und Beinen ausüben soll. In Gruppe B ( $n=49$ Beine) kam der Struva $35{ }^{\circledR}$ zum Einsatz mit einem angegebenen Druck von $35 \mathrm{mmHg}$ an den Knöcheln. Beide Strümpfe hatten eine Taillenbefestigung und waren an den Zehen offen. Die Kompressionsstrümpfe wurden an den ersten 7 Tagen 24 Stunden lang getragen. Am 3. postoperativen Tag erfolgte eine telefonische Erfragung postoperativer Symptome. Am 7. postoperativen Tag erfolgte eine klinische Untersuchung mit Entfernung der Wundversorgung und der Kompression. In den folgenden Tagen wurden tagsüber Kompressionsstrümpfe oder -strumpfhosen mit einem Knöcheldruck von 18-21 mmHg getragen. Die Nachuntersuchungen erfolgten am 7. und 40. postoperativen Tag mit Erfragung der Compliance und Beurteilung der Haut. Zur Beurteilung der Ödementwicklung wurde eine Bioimpedanzspektrosko- 
pie mit dem L-Dex U400 ${ }^{\circledR}$ (ImpediMed, Australien) System durchgeführt.

Die Nachuntersuchungen konnten an allen Beinen durchgeführt werden. Am 3. und 7. postoperativen Tag waren die meisten Symptome nach der Kompressionsbehandlung mit Struva 35 in Gruppe B geringer als in Gruppe A. Insbesondere wurden geringere Schmerzen und ein geringeres Schweregefühl angegeben. Dies galt ebenso am 40 . postoperativen Tag. Am 7. postoperativen Tag ergaben sich für Gruppe B signifikant bessere Werte für verschiedene Parameter. Das galt für das Umhergehen $(p=0,046)$, die Stabilität und Tolerabilität der Kompressionsstrümpfe ( $p=0,021$ und 0,060$)$ sowie für die Wundheilung $(p=0,010)$. Nach den
Ergebnissen der Bioimpedanz führte die Behandlung mit den Kompressionsstrümpfen mit $35 \mathrm{mmHg}$ zu einer leicht verbesserten Abnahme des Ödems. Die Bioimpedanzparameter nahmen am 7. Tag in beiden Gruppen zu und am 40 Tag in beiden Gruppen ab. In Gruppe B verbesserte sich die Reaktanz der Beine und der L-Dex am 7. $(p=0,039)$ und 40. $(p=0,012)$ Tag. Die Farbduplexsonografie am 40. Tag ergab in der gesamten Kohorte keine Hinweise auf tiefe oder oberflächliche Thrombosen. Relevante Komplikationen traten während des Studienzeitraums nicht auf.
FAZIT

Nach Einschätzung von Cavezzi et al. zeige ihre Studie, dass eine Kompressiontherapie mit $23-35 \mathrm{mmHg}$ nach Schaumsklerosierung und Phlebektomie effektiv und sicher ist. In der unmittelbaren und frühen postoperativen Phase zeigte sich ein positiver Effekt auf die Gewebezusammensetzung und die Heilung. Außerdem habe sich eine leichte Überlegenheit bei der Anwendung von Kompressionsstrümpfen mit $35 \mathrm{mmHg}$ gezeigt.

Dr. Gabriele Dobler, Berlin 